\title{
THEMIS observations of compressional pulsations in the dawn-side magnetosphere: a case study
}

\author{
G. I. Korotova ${ }^{1,2}$, D. G. Sibeck ${ }^{3}$, V. Kondratovich ${ }^{4}$, V. Angelopoulos ${ }^{5}$, and O. D. Constantinescu ${ }^{6}$ \\ ${ }^{1}$ IZMIRAN, Troitsk, Moscow Region, Russia \\ ${ }^{2}$ IPST, UMD, College Park, MD, USA \\ ${ }^{3}$ Code 674, NASA/GSFC, Greenbelt, MD, USA \\ ${ }^{4}$ SP Systems, Greenbelt, MD, USA \\ ${ }^{5}$ IGPP/ESS, UCLA, Los Angeles, CA, USA \\ ${ }^{6}$ Institut für Geophysik und extraterrestrische Physik, Technische Universität Braunschweig, Mendelssohnstrasse 3, \\ Braunschweig, Germany
}

Received: 5 May 2009 - Revised: 9 September 2009 - Accepted: 11 September 2009 - Published: 2 October 2009

\begin{abstract}
We present THEMIS-A low- and high-energy plasma, magnetic field, and energetic particle observations of long period (11-36 min) irregular compressional pulsations in the dawnside magnetosphere from 08:00 to 12:24 UT on 7 November 2007. We demonstrate that the pulsations maintain thermal and magnetic pressure balance, then employ finite gyroradius techniques to determine wave properties from the gyrophase distributions of $5-10 \mathrm{keV}$ ions. The waves generally move sunward at velocities $\sim 10 \mathrm{~km} \mathrm{~s}^{-1}$ with the background plasma convection flow. Wavelengths range from 6700 to $23300 \mathrm{~km}$, corresponding to azimuthal wavenumbers $\mathrm{m}$ from 18 to 76 . Wave periods decrease with increasing radial distance. Having determined the parameters describing the waves, we consider three previously proposed explanations: generation by substorm injection, generation by bounce or drift-bounce instabilities, and generation by the drift-mirror instability. The interval was quiet geomagnetically, arguing against any relationship to substorm injections. We found that ions with low energies of 69$628 \mathrm{eV}$ or high energies of $28-615 \mathrm{keV}$ would have been required to account for drift-bounce resonance during this interval, but inspection reveals ion fluxes at these energies near background levels during the time period considered. On the other hand, the criteria for the drift mirror instability are marginally satisfied. As predicted for the drift mirror instability, particle distributions peak more sharply near $90^{\circ}$ pitch angles during magnetic field strength enhancements than during strength depressions. At this point we therefore interpret the compressional pulsations observed by THEMIS A in terms of the drift mirror instability.
\end{abstract}

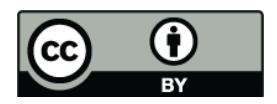

Correspondence to: G. I. Korotova (gkorotov@umd.edu)
Keywords. Ionosphere (Wave-particle interactions) - Magnetospheric physics (Energetic particles, precipitating; MHD waves and instabilities)

\section{Introduction}

Compressional pulsations with periods ranging from 100 to more than $600 \mathrm{~s}$ are common in the equatorial morning and afternoon magnetosphere at distances beyond $L=8$ (e.g., Baumjohann et al., 1987; Takahashi et al., 1990a; Anderson et al, 1990; Zhu and Kivelson, 1991; Vaivads et al., 2000, 2001) when the plasma $\beta$ is large (Zhu and Kivelson, 1994; Rae et al., 2007). At dusk they occur during geomagnetically disturbed intervals, are associated with plasma injections, and propagate westward (sunward) at the $10-20 \mathrm{~km} \mathrm{~s}^{-1}$ gradient-curvature drift velocities expected for 10-30 keV ions (Barfield and McPherron, 1972; Takahashi et al., 1985). At dawn they occur during quiet intervals and move eastward (sunward) with phase velocities close to the plasma convection velocity (Vaivads et al., 2001). Wave periods may (Baumjohann et al., 1987; Constantinescu et al., 2009) or may not (Woch et al., 1990) increase with increasing radial distance, corresponding to decreasing magnetic field strength.

Because the pulsations modulate the flux of suprathermal particles (e.g., Kokubun, 1977), finite gyroradius techniques can be used to sense the motion of wave fronts past the observing spacecraft, determine propagation velocities in the plane perpendicular to the ambient magnetic field, and then determine wavelengths and wave numbers (Su et al., 1977). Case and statistical studies reveal that the waves typically exhibit wavelengths from 2000 to $7000 \mathrm{~km}$ and therefore wave

Published by Copernicus Publications on behalf of the European Geosciences Union. 
numbers $\mathrm{m}$ from 15 to 120 (e.g., Takahashi et al., 1985, 1990a; Zhu and Kivelson, 1991; Lessard et al., 1999; Vaivads et al., 2001).

Three causes for the compressional pulsations have been proposed: drift mirror instabilities due to pressure anisotropies (Hasegawa, 1969; Pokhotelov et al., 1985; Cheng and Lin, 1987), drift-bounce instabilities (Southwood, 1976; Chen and Hasegawa, 1991), and the injection of blobs of plasma sheet plasma into the inner magnetosphere (Haerendel et al., 1999).

The drift mirror instability occurs spontaneously in inhomogeneous high $\beta$ plasmas ( $\beta$ is the ratio of plasma pressure to magnetic pressure) when the temperature perpendicular to the magnetic field exceeds that in the parallel direction. By extending the theory for the mirror instability (Chandrasekhar et al., 1958) to include gradients in the magnetic field $B$ and the hot plasma density $n$, a coexisting cold plasma, and the effect of finite cyclotron radius, Hasegawa (1969) showed that the growing mode becomes oscillatory (in space) via coupling with drift waves produced by $\Delta B$ and $\Delta n$. The fluid dynamic criterion for the mirror instability is:

$\Gamma=1+\beta_{\perp}\left[1-T_{\perp} / T_{I I}\right]<0$,

where $\beta_{\perp}=\left(n_{i} k T_{i \perp}+n_{e} k T_{e \perp}\right) /\left(B^{2} / 2 \mu_{0}\right), T_{I I, \perp}$ - plasma temperature parallel and perpendicular to the ambient magnetic field, $\mu_{0}$ - permeability of free space.

Magnetic field strength enhancements should energize particles and squeeze out those with low pitch angles, resulting in normalized particle fluxes that peak more narrowly about $90^{\circ}$ pitch angles during field strength enhancements than during field strength depressions (Southwood and Kivelson, 1993).

By contrast, Southwood et al. (1969) proposed that high$\mathrm{m}$ waves would be excited by a bounce-drift resonant interaction with the energetic particles trapped in the magnetosphere. In the dipole regions of the magnetosphere the mirror magnetic field geometry causes ions and electrons to execute a bouncing motion back and forth along the field and also execute a slow drift in longitude across the field due to the magnetic gradient and curvature drift. If the wave field varies as $\exp (\operatorname{im} \phi-i \omega t)$ in longitude $\phi$, and time $t$, resonance can occur if

$\omega-m \omega_{d}-N \omega_{b}=0$

where $\omega_{d}$ and $\omega_{b}$ are the angular drift and bounce frequencies, $N$ is an integer, and $m$ is the azimuthal wave number. The particles that satisfy this resonance condition see a constant wave electric field so the wave will either grow or damp depending on the structure of the particle distribution and the energy availability. Finite pressure and inhomogeneity introduce a coupled compressional signal (Southwood and Kivelson, 1997). Making considerable simplifications, particularly neglecting harmonics, Southwood et al. (1969) showed that the resonance condition including the Doppler shift due to the East-West particle drift is a quadratic for the particle energy. There is a low energy for which the bounce frequency almost equals the wave frequency and a high energy for which the Doppler shifted frequency is much higher than the wave frequency.

The symmetry of the wave field about the equator determines which resonances are important (see, e.g., Southwood et al., 1976). A disturbance with symmetrical (antisymmetrical) displacement $\xi$ about the equator will only resonate with particles in even (odd) $N$ resonances. Southwood et al. (1969) showed that the $N= \pm 1$ resonances should dominate in the magnetosphere and that the most easily excited waves should be antisymmetric about the equator. They also showed that the resonant particle contribution to wave growth or damping depends on the sign of $d f / d W$, where $f$ denotes phase space density and $W$ stands for particle kinetic energy. The instability occur when there is a sufficient spatial gradient in some part of the resonant distribution or if the plasma velocity distribution is inverted at some point such that $d f / d W>0$ (Southwood and Hughes, 1983). Southwood (1973) predicted that particle flux oscillations associated with the wave just above the resonant energy should be $180^{\circ}$ out of phase with those just below.

Haerendel et al. (1999) reported the frequent occurrence of azimuthally-limited, asymmetric, high-beta $(1<\beta<10)$ plasma blobs outside $\sim 9 R_{E}$ in the equatorial post-midnight to early morning magnetosphere. They indicated that the sunward-drifting blobs might result from some sort of mirror waves instability, and attributed the source of the plasma in the blobs to the morningside boundary layer at distances further tailward. However, Haerendel et al. (2004) noted similar events in Cluster observations much further $\left(\sim 19 R_{E}\right)$ from Earth in the post-midnight magnetotail. They then attributed both sets of events to the pulsed earthward transport of blobs originating in the plasma sheet during the recovery and postrecovery phases of substorms.

Due to the antiphase relationship between the density/pressure and the magnetic field strength, the waves might also be interpreted in terms of slow mode MHD waves. However, as noted by Su et al. (1980), slow mode waves damp heavily in homogenous plasma when ion temperatures exceed those for electrons, as in the example presented here. Consequently we do not consider this possibility further.

This paper employs high-time resolution THEMIS-A magnetic field and plasma observations to determine the characteristics of and test proposed mechanisms for compressional pulsations in the dawnside magnetosphere observed from 08:00 to 12:24 UT on 7 November 2007.

\section{Instrumentation}

The ESA electrostatic analyzer on the THEMIS spacecraft measures the distribution functions of 0.005 to $25 \mathrm{keV}$ ions and 0.005 to $30 \mathrm{keV}$ electrons over $4 \pi$-str and provides 
accurate $3 \mathrm{~s}$ time resolution plasma moments, pitch angle and gyrophase particle distributions (McFadden et al., 2008). The SST solid-state telescopes, each comprising two sensors, measure the superthermal $(0.02-1 \mathrm{MeV})$ parts of the ion and electron distributions over $3 \pi$-str (Angelopoulos, 2008). Neither the ESA nor the SST distinguish between ion species. The FGM triaxial fluxgate magnetometer measures the background magnetic field and its low frequency fluctuations up to $64 \mathrm{~Hz}$ (Auster at el., 2008). Throughout most of their orbits, the spacecraft operate in slow survey mode, returning magnetic field vectors, plasma moments calculated on board, and spectra with 3s time resolution, but full ESA and SST particle distribution functions only once every several minutes. During fast survey mode, the spacecraft also return ESA and SST particle distribution functions with reduced angular resolution every $3 \mathrm{~s}$. Full or reduced distribution functions are needed to calculate temperature and pressure anisotropies taking into account the spacecraft potential.

\section{Orbits}

Figure 1 presents the trajectories of the 5 THEMIS spacecraft and GOES-11/12 from 08:00 to 12:24 UT on 7 November 2007. The locations of the bow shock and magnetopause have been scaled (for pressure $=1.13 \mathrm{nPa}$ and $B_{z}=0 \mathrm{nT}$ ) in a self-similar manner from the positions expected for typical solar wind parameters to account for the fact that THEMIS$\mathrm{B}$ was in the solar wind, - $\mathrm{C}$ in the magnetosheath, and the remaining spacecraft were in the equatorial dawn magnetosphere. Each of the spacecraft moved sunward along its trajectory during this interval. In particular, THEMIS-A moved radially outward and slightly sunward inside the magnetosphere from GSM $(\mathrm{X}, \mathrm{Y}, \mathrm{Z})=(-4.7,-8.7,1.0)$ to $(\mathrm{X}, \mathrm{Y}$, $\mathrm{Z})=(-3.3,-12.6,1.8) R_{E}$.

\section{Observations}

The interval from 08:00 to 12:30 UT on 7 November 2007 was very quiet geomagnetically, occurring in the midst of a prolonged interval during which $K_{p}$ was 0 . As illustrated in the top panel of Fig. 2, which shows dynamic pressures calculated from slow mode distribution functions, neither THEMIS-B (in the solar wind) nor THEMIS-C (in the magnetosheath) observed any significant variation in the solar wind/magnetosheath dynamic pressure. The solar wind and magnetosheath magnetic fields were generally northward prior to 10:28 UT, but southward thereafter. Geosynchronous orbit was also quiet. As illustrated by the 1 min time resolution GOES-11 and -12 magnetic field GSM latitudes in the third panel of Fig. 2, neither spacecraft observed any evidence for magnetic field line stretching and subsequent dipolarization. None of the LANL geosynchronous spacecraft observed plasma or energetic particle injections (not shown).
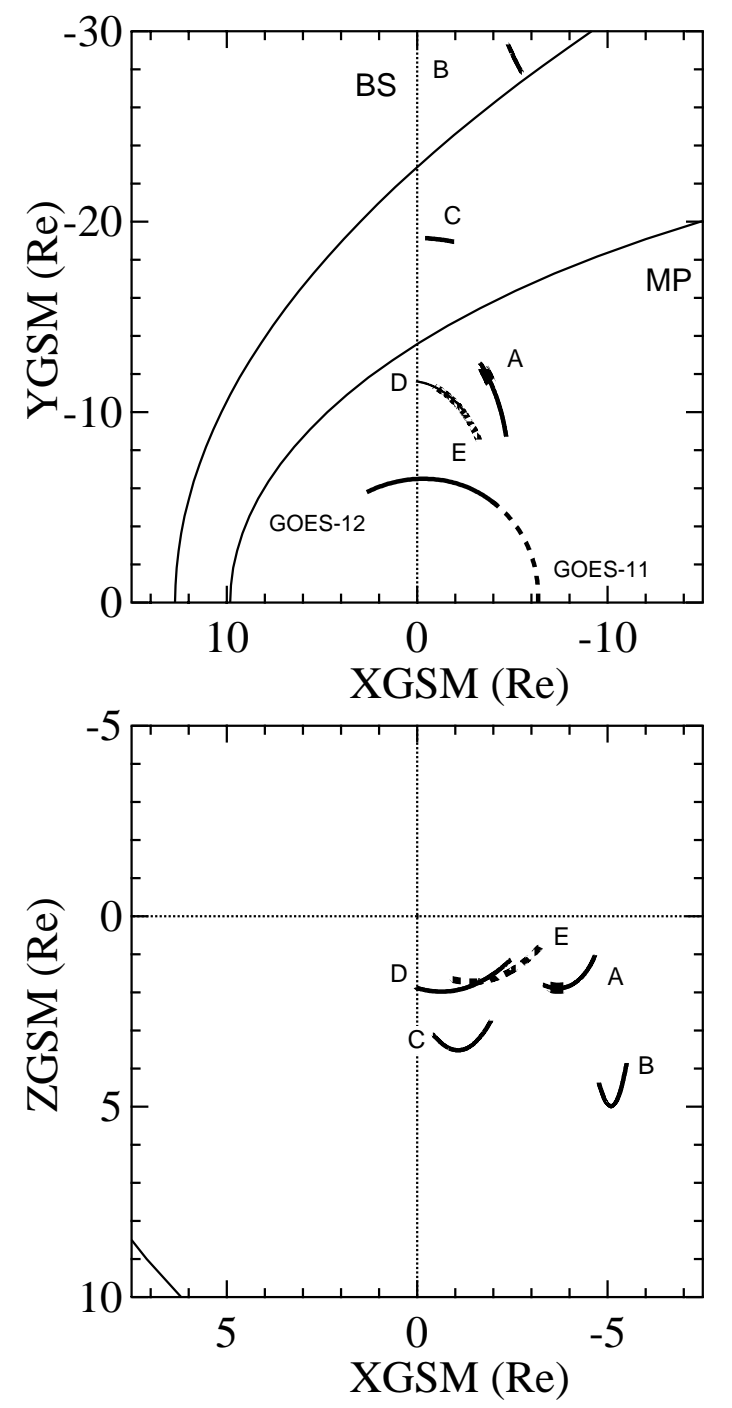

Fig. 1. Locations of THEMIS-A, B, C, D, E and GOES-11/12 in the GSM X-Y and X-Z planes from 08:00 UT to 12:24 UT on 7 November 2007. Grey shading marks the portion of the THEMIS-A orbit from 11:00 UT to 12:15 UT. The locations of the bow shock and magnetopause were chosen to place the spacecraft in the observed regions of geospace during this interval.

However, the interval was not entirely quiet. Very high latitude ground magnetograms, shown in the fourth and fifth panels of Fig. 2 provide evidence for two weak high-latitude substorms during this interval. High latitude stations Barrow (geomagnetic latitude $69.8^{\circ}$, geomagnetic longitude $248.8^{\circ}$ ), Kaktovik $\left(70.7^{\circ}, 257.8^{\circ}\right)$, and Inuvik $\left(70.9^{\circ}, 271.1^{\circ}\right)$ observed $\sim 100 \mathrm{nT}$ negative $\mathrm{X}$ bays (i.e. southward deflections) indicative of dawn to dusk ionospheric currents in substorm current wedges from 10:00 to 10:20 and 11:10 to 11:30 UT. The longitudinal extent of these substorm current wedges was no more than $40^{\circ}$ or three hours in local time: Shumagin $\left(54.3^{\circ}, 256.7^{\circ}\right)$ observed eastward magnetic field deflections 


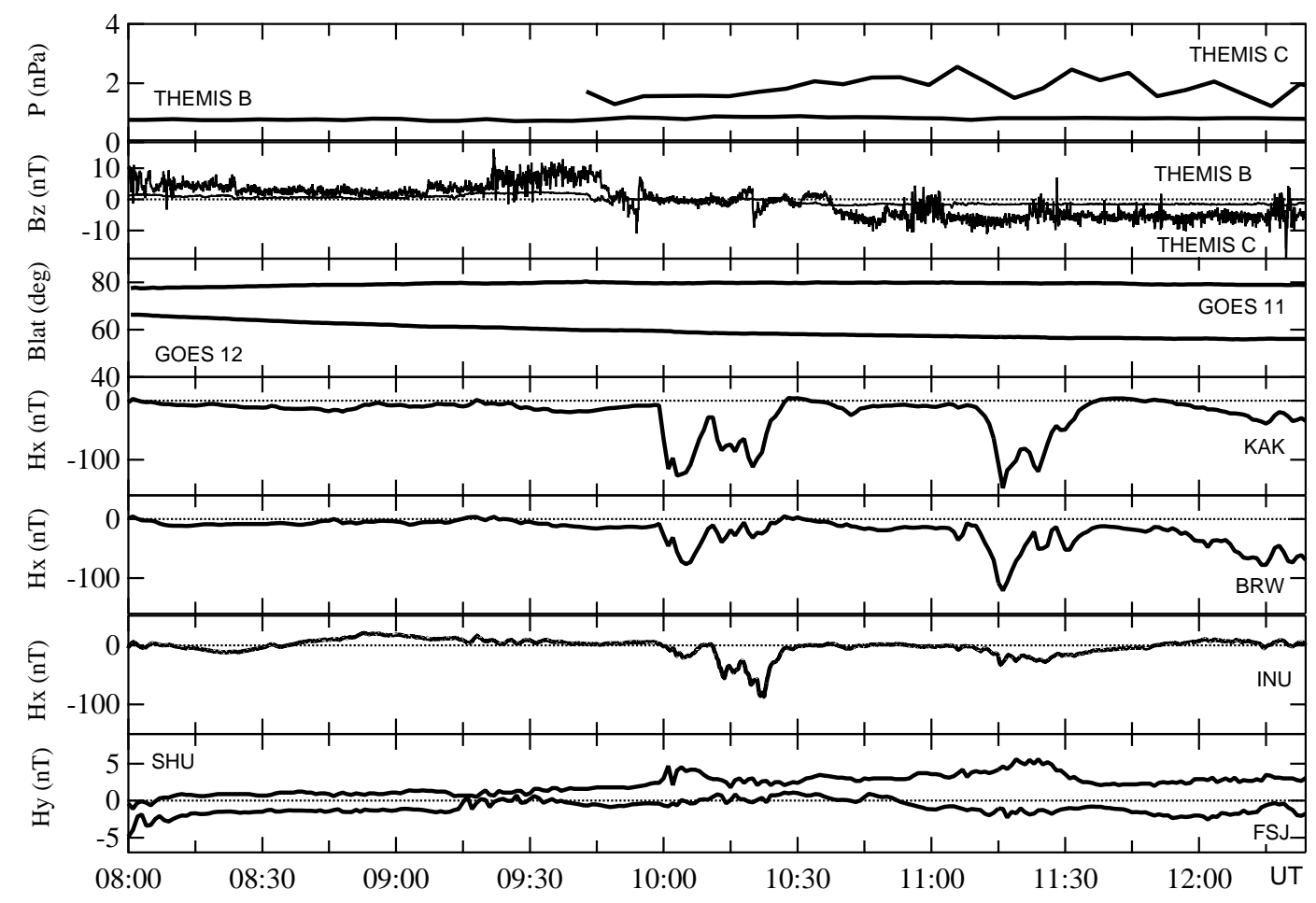

Fig. 2. From top to bottom: THEMIS-B/C solar wind/magnetosheath dynamic pressure, THEMIS-B/C Bz magnetic field component, GOES-11/12 magnetic field latitudes, and high latitude ground magnetometer $\mathrm{X}$ components.

indicating a location equatorward from the upward current, while Fort St. John $\left(62.0^{\circ}, 296.6^{\circ}\right)$ observed westward magnetic field deflections indicating a location equatorward from the downward current. Furthermore, Geotail, located at GSM $(\mathrm{x}, \mathrm{y}, \mathrm{z})=(-9.6,5.8,-3.2) R_{E}$ in the evening sector, observed a nondispersive burst of energetic $(E=40-100 \mathrm{keV})$ ions from 11:10 to 11:35 UT (not shown).

Although THEMIS-A, -D, and -E observed compressional pulsations in the dawnside magnetosphere for many hours on 7 November 2007, this paper focuses on the reduced distributions functions returned each $3 \mathrm{~s}$ during fast survey mode observations by THEMIS-A from 08:00 UT to 12:24 UT. From top to bottom, Fig. 3 presents THEMIS-A observations of the magnetic field and ion flow velocity in GSM coordinates. The magnetic field strength decreased slowly from 25 to $15 \mathrm{nT}$ as THEMIS-A moved outward, while the sunward component of the convection velocity $\left(V_{x}\right)$ increased from 2 to $13 \mathrm{~km} \mathrm{~s}^{-1}$ and the total convection velocity ( $\left.V_{\text {tot }}\right)$ increased from 13 to $24 \mathrm{~km} \mathrm{~s}^{-1}$.

From 08:00 to 10:00 UT, THEMIS-A observed weak irregular variations in the magnetic field strength and direction with "periods" of $30 \mathrm{~min}$ and amplitudes of $\sim 5 \mathrm{nT}$ and apparently unrelated plasma flow variations with periods from 5 to 7 min and amplitudes $\sim 5 \mathrm{~km} \mathrm{~s}^{-1}$. The mean convection velocity was very small $\left(V_{x}=2.5 \mathrm{~km} \mathrm{~s}^{-1}\right)$. From 10:00 to 11:00 UT, the magnetic field exhibited more regular pulsations with periods of $20-25 \mathrm{~min}$ and amplitudes
$10 \mathrm{nT}$. North/south plasma velocity variations on the order of $10 \mathrm{~km} \mathrm{~s}^{-1}$ were superimposed upon a $7 \mathrm{~km} \mathrm{~s}^{-1}$ sunward background convection velocity. From 11:00 UT to 12:15 UT there were regular compressional oscillations in the magnetic field with in phase oscillations of all three components, periods of 13-15 min, and peak-to-peak amplitudes of 13-14 nT. The amplitude of the corresponding flow variations was $\sim 15 \mathrm{~km} \mathrm{~s}^{-1}$, superimposed on a mean sunward convection of $\sim 13 \mathrm{~km} \mathrm{~s}^{-1}$.

The weak second harmonic seen in the $B_{z}$ component and total magnetic field strength during this interval resembles that previously reported at geosynchronous orbit by Coleman (1970), namely the magnetic perturbation of the compressional component oscillates with a frequency twice as high as that the transverse component. Zhu and Kivelson (1994) found numerous such events in the dawnside and duskside magnetosphere beyond $9 R_{E}$ which Takahashi et al. (1987a) interpreted as harmonic structures in a model for an antisymmetric standing wave. We defer detailed discussion of THEMIS observations of this phenomenon to a subsequent paper.

Figure 4 presents further ESA plasma and FGM magnetic field parameters characterizing the pulsations. From top to bottom, the figure shows the ion and electron densities, the ion and electron temperatures perpendicular and parallel to the magnetic field, the magnetic, thermal, and total pressures, and (for discussion later) results from a test of the mirror 
THEMIS A

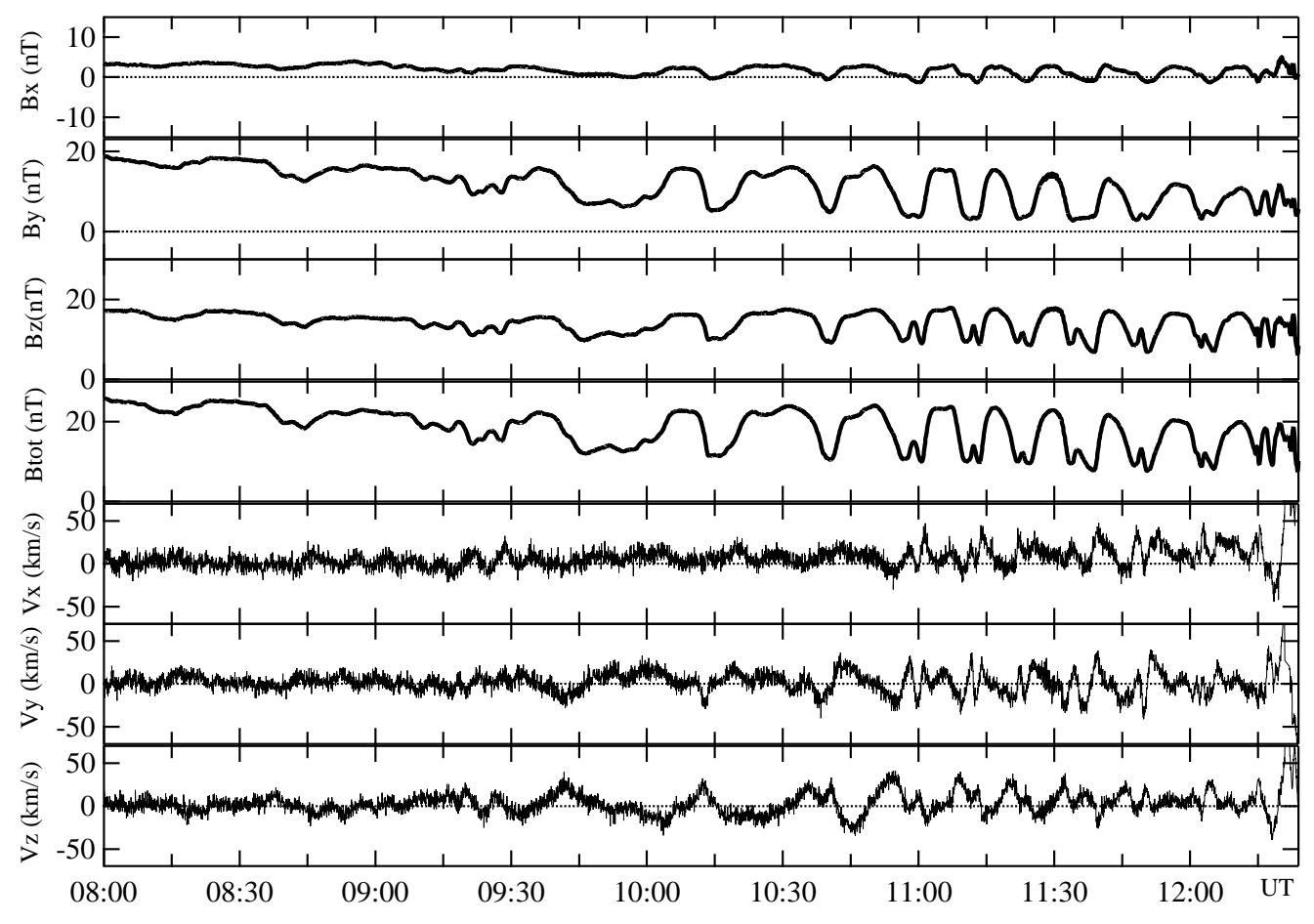

Fig. 3. THEMIS-A observations of the magnetic field and ion velocity from 08:00 to 12:24 UT on 7 November 2007 in GSM coordinates.

instability criterion. Ion temperatures are anisotropic, with greater temperatures perpendicular than parallel to the magnetic field. Although small, the nearly isotropic electron temperatures are not negligible $\left(T_{e} \sim 0.2 T_{i}\right)$, as has sometimes been assumed. Their contribution must be considered when calculating the total thermal pressure, which oscillates in antiphase with the magnetic field pressure so as to keep with the sum nearly constant (e.g., Zhu and Kivelson, 1991).

Figures 5 and 6 show THEMIS-A ion and electron energy spectra in the energy ranges from $2 \mathrm{eV}$ to $25 \mathrm{keV}$ (ESA) and $25 \mathrm{keV}$ to $150 \mathrm{keV}$ (SST). Ion fluxes peak at energies from 2 to $10 \mathrm{keV}$, while electron fluxes peak from $\sim 500 \mathrm{eV}$ to $5 \mathrm{keV}$. The compressional pulsations modulate the fluxes of both species at all energies, particularly after 09:40 UT when the pulsations exhibited greater amplitudes. The SST observed two weak electron injections with energies from 25 to $70 \mathrm{keV}$ at 10:10 UT and 11:30 UT about $10 \mathrm{~min}$ after each of the two substorm bays noted in the high-latitude ground magnetograms. THEMIS-A observed no corresponding energetic ion injections.

\section{Wave characteristics}

From top to bottom, Fig. 7 presents THEMIS-A ESA 5$10 \mathrm{keV}$ ion pitch angle distributions, the same ion pitch angle distributions normalized to the peak flux at each instant, 5$10 \mathrm{keV}$ ion gyrophase distributions integrated over $45-135^{\circ}$ pitch angles, the FGM magnetic field strength, and the ESA ion number density moment from 11:00 UT to 11:30 UT. Ion pitch angle distributions peak near $90^{\circ}$, as is typical during compressional Pc5 waves (Kremser et al., 1981). Both the plasma density (primarily cold particles) and the flux of 5-10 keV ions vary in antiphase with the magnetic field strength. Since particles with $0^{\circ}$ phase angles are moving duskward $(+\mathrm{Y})$ and those with $90^{\circ}$ phase angles are moving antisunward $(-\mathrm{X})$, the fact that increases and decreases in particle flux first appear at $180^{\circ}$ phase angles and only later ( $\Delta T=90$ to $180 \mathrm{~s}$ ) at $0^{\circ}$ phase angles in the presence of a northward magnetospheric magnetic field indicates sunwardmoving wavefronts.

For comparison with the predictions of the three proposed models, we can determine the proton gyroradius $r_{\text {gyro }}$, the azimuthal wavefront velocity $V_{d r}$, the wavelength $\lambda$, and the azimuthal wave number $m$ from the observed gyrophase lags $\Delta T$ and wave periods $T$ using the following formulae:

$$
\begin{aligned}
& r_{\text {gyro }}=\left(2 m_{p} E / q\right)^{1 / 2} / B \\
& V_{d r}=2 r_{\text {gyro }} / \Delta T \\
& \lambda=V_{d r} T \\
& m=2 \pi R_{s c} / \lambda
\end{aligned}
$$

where $R_{s c}$ is the radial distance of the spacecraft from Earth, $m_{p}$ is the mass of a proton, $B$ is the observed magnetic field 
THEMIS A

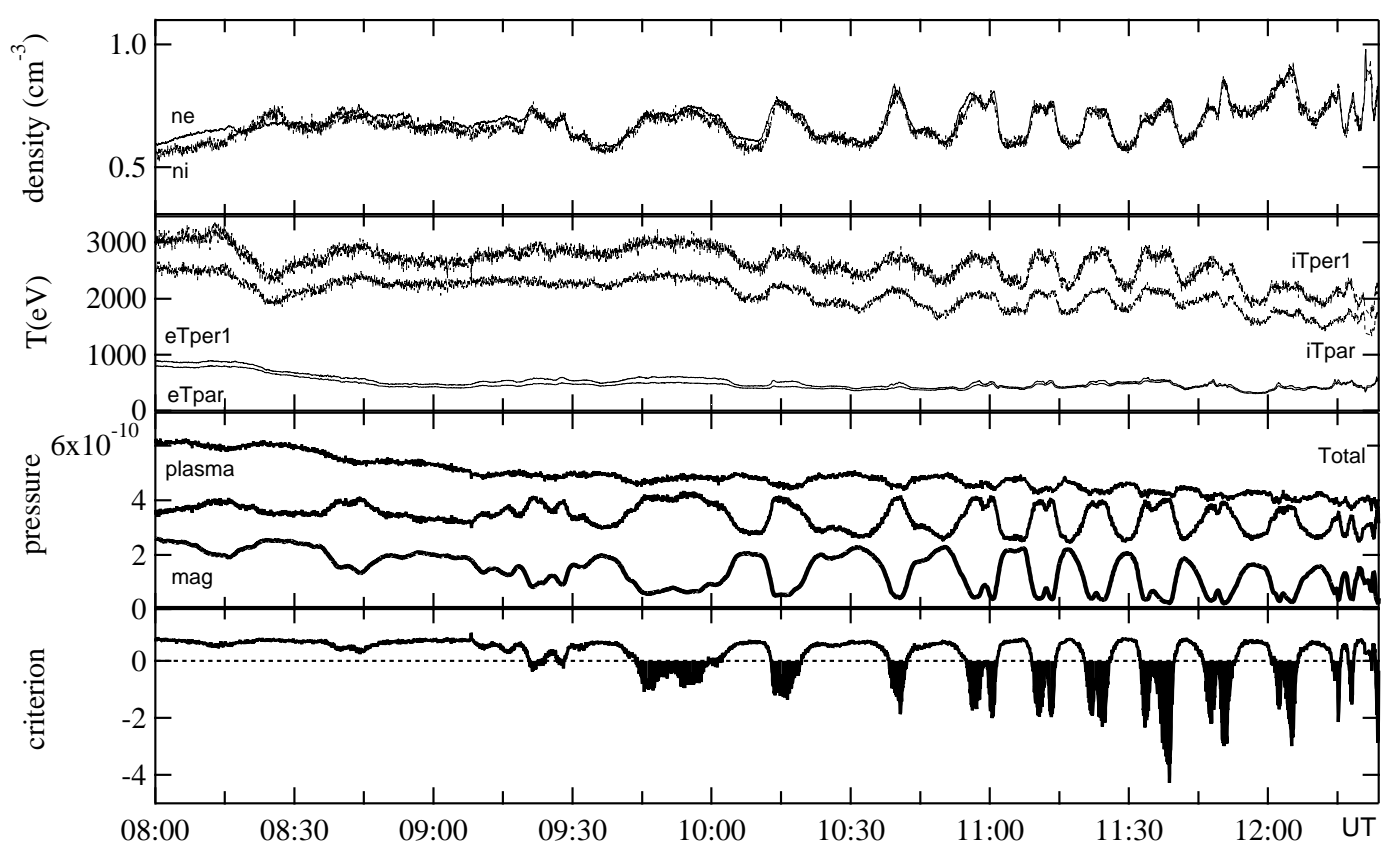

Fig. 4. Plasma parameters for THEMIS-A from 08:00 UT to 12:24 UT on 7 November 2007. From top to bottom, the panels show (a) the ion and electron densities, (b) the ion and electron temperatures perpendicular and parallel to the magnetic field, (c) the magnetic pressure $\left(B^{2} / 2 \mu_{0}\right)$, the sum of the ion and electron $\left(n_{i} k T_{i \perp}+n_{e} k T_{e \perp}\right)$ thermal pressures perpendicular to the magnetic field, and sum of the plasma and magnetic pressures, and (d) results from test of the mirror instability criterion. Shaded grey areas indicate the times where the drift mirror instability is satisfied $(<0)$.

strength, $q$ is the charge of a proton, and $E$ is the energy of the particles (taken as the geometric mean for this energy channel or $7 \mathrm{keV}$ here). Figure 8 presents the results of these calculations for the full interval from 08:00 UT to 12:24 UT, but plotted as a function of radial distance from Earth. Pulsation "periods" slowly decrease from more than $30 \mathrm{~min}$ at $10.5 R_{E}$ to values ranging from 13 to $15 \mathrm{~min}$ at $12.3 R_{E}$ and beyond. Gyroradii increase from 600 to $800 \mathrm{~km}$. The velocity of the waves inferred from the finite gyroradius technique is $\sim 10 \mathrm{~km} \mathrm{~s}^{-1}$ sunward.

Finally, wavelengths ranged from values greater than $10000 \mathrm{~km}$ near Earth to values less than $10000 \mathrm{~km}$ further away, resulting in azimuthal wave numbers ranging from less than 40 near Earth to almost 80 further away.

\subsection{Test of three proposed models}

In this section, we test the predictions of the drift-bounce, plasma blob, and drift-mirror model predictions for wave generation on 7 November 2007.

\subsection{Drift bounce resonance test}

The drift-bounce model predicts the existence of a particle population whose bounce and drift frequencies resonate with and provide energy to compressional waves in the magneto- sphere. The phase relationship between particle fluxes and the wave should vary $180^{\circ}$ across the resonant frequency.

To obtain the energy of resonant protons required for drift bounce resonance for low frequency waves we solve a quadratic for the resonance velocities on a given field line (Takahashi et al., 1990b):

$$
\begin{aligned}
& \omega_{b}^{2}-\left(m \omega_{d}-\omega_{r}\right)^{2}=0 \\
& \left(\omega_{b}-m \omega_{d}+\omega_{r}\right)\left(\omega_{b}+m \omega_{d}-\omega_{r}\right)=0
\end{aligned}
$$

We use the observed wave frequencies for $\omega_{r}$. Drift $\omega_{d}$ and bounce frequencies $\omega_{b}$ are determined from the formula of Hamlin et al. (1961) for a given equatorial pitch-angle, energy, and L-value.

$$
\begin{aligned}
& \omega_{d}=\left(3 m_{p} V^{2} R_{s c} P(\alpha)\right) /\left(q B_{0} R_{E}^{2}\right), \\
& \omega_{b}=\pi V /\left(2 R_{E} R_{s c} T(\alpha)\right), \\
& T(\alpha)=1.30-0.56 \sin \alpha, \\
& P(\alpha)=0.35+0.15 \sin \alpha, \alpha=45^{\circ}
\end{aligned}
$$

Then the energy $W$ of the resonant protons can be determined from $W=1 / 2\left(m_{p} V^{2} / q\right)$. The studies of e.g. Takahashi et al. (1987b) and Zhu and Kivelson (1994) showed that the magnetic equator is a node for the compressional component 


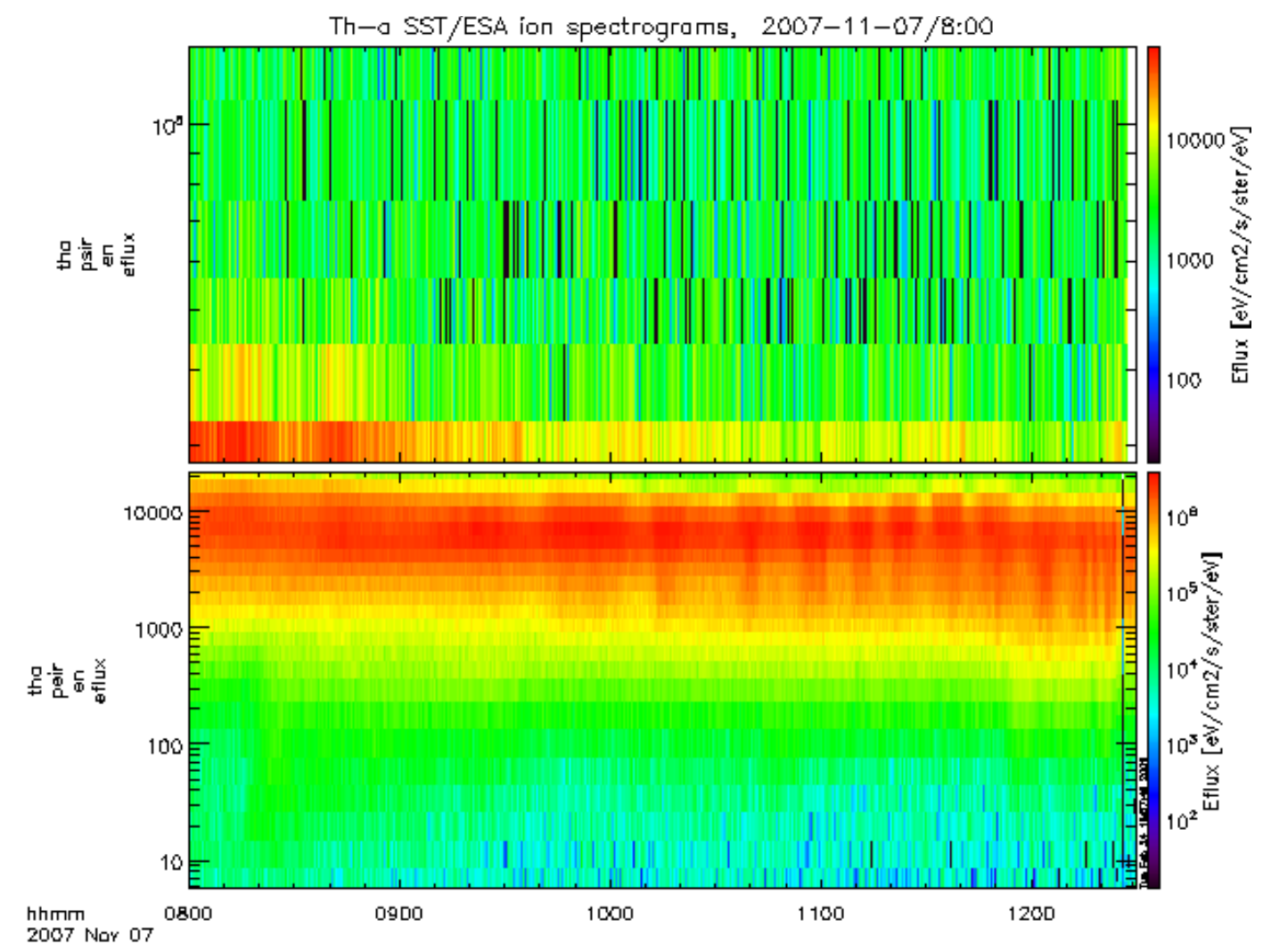

Fig. 5. Energy flux spectrograms for ions in the range of energies from $2 \mathrm{eV}$ to $25 \mathrm{keV}$ (ESA) and from $25 \mathrm{keV}$ to $150 \mathrm{keV}$ (SST) on 7 November 2007 for THEMIS-A.

and antinode for the transverse component, i.e. the pulsations are antisymmetric with respect to the.equator (second harmonic). Southwood and Kivelson (1981) showed mathematically that in the case where a signal electric field is antisymmetric about the equator, resonance is possible only for particles that detect odd harmonics of the bounce frequency. Therefore, we set $N= \pm 1$ and the values for $m$ derived using the finite gradient techniques above as a function of radius. The bottom panel of Fig. 8 shows that the proton energy required for the high-energy resonance was typically 28 to $75 \mathrm{keV}$, but ranged as large at $615 \mathrm{keV}$. We obtained that the proton energies required for the low-energy resonance ranging from $69-628 \mathrm{eV}$.

Inspection of Fig. 5 provides no evidence for significant particle fluxes at either the high energies greater than $28 \mathrm{keV}$ or the low energies from $69-628 \mathrm{eV}$ required for resonance with the compressional waves. Nor does the figure provide any evidence for a reversal in the phase relationship between particles fluxes across these resonant energies. We therefore rule out the bounce drift resonance as a possible candidate for generation of the compressional waves.

\subsection{Criterion for plasma blob convection}

The compressional pulsations may correspond to high $\beta$ plasma blobs injected by geomagnetic substorms. If so, the pulsations should begin, or attain greater amplitudes, when enhanced ion fluxes arrive shortly after the onset of the two weak substorms observed in ground magnetograms at 10:00 and 11:10 UT. Although Fig. 6 indicates that dispersed injections of enhanced electron fluxes arrive at 10:10 and 11:30 UT, Fig. 5 provides no evidence for any corresponding increases in the ion fluxes. Figure 4 indicates that neither peak nor minimum densities associated with the waves vary significantly at these times. Figure 3 demonstrates that convection velocities increase gradually throughout the interval from 08:00 to 12:24 UT, rather than abruptly at the substorm onset times. We therefore conclude that the wave activity is not associated with the arrival of drifting clouds of ions injected by substorms.

\subsection{Mirror instability test}

We expect plasma and magnetic field conditions observed during drift mirror mode waves to satisfy the specified instability criteria, the waves to propagate through the ambient plasma at the ion gradient curvature drift velocity, and ion 


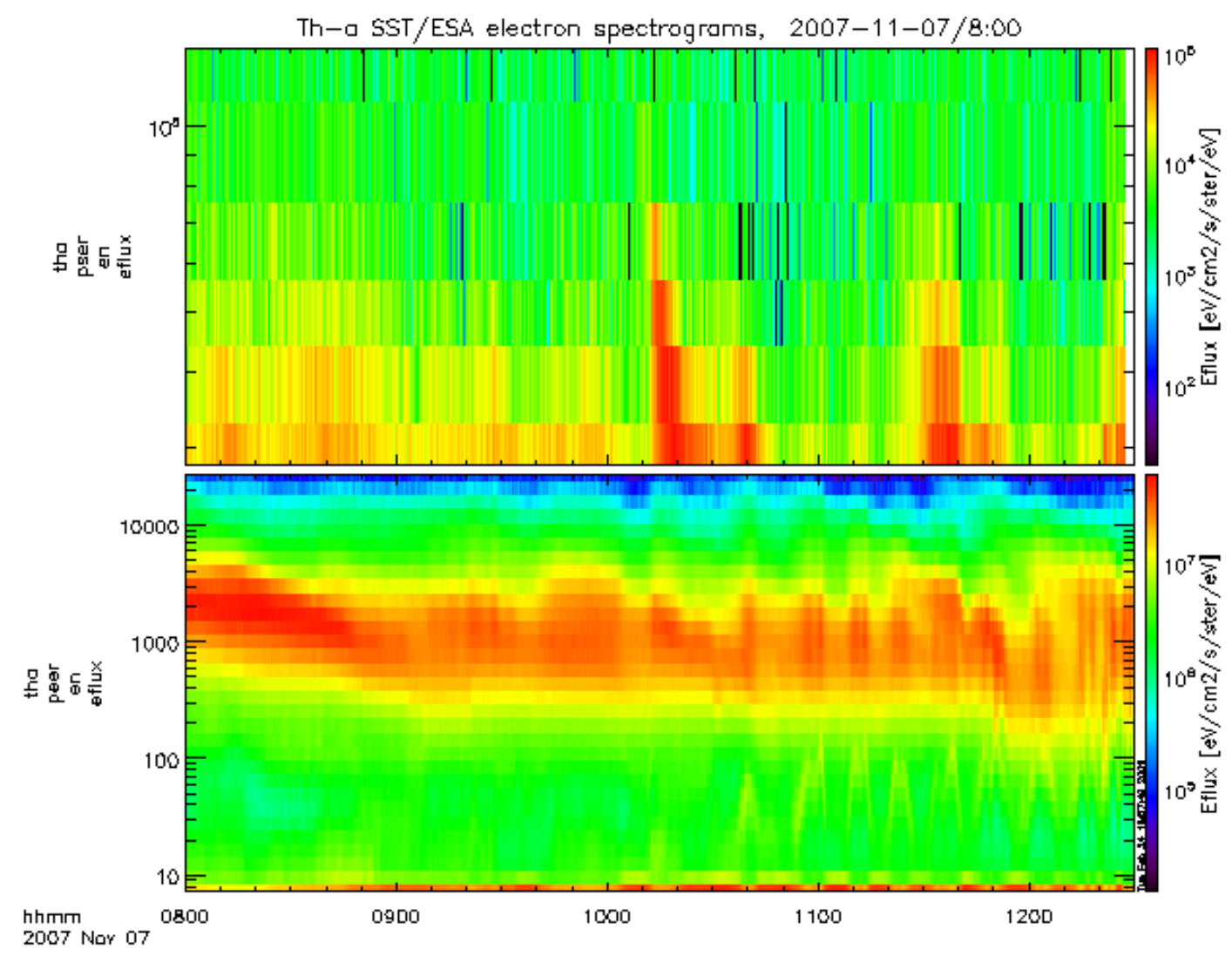

Fig. 6. Energy flux spectrograms for electrons in the range of energies from $2 \mathrm{eV}$ to $25 \mathrm{keV}$ (ESA) and from $25 \mathrm{keV}$ to $150 \mathrm{keV}$ (SST) on 7 November 2007 for THEMIS-A.

pitch angle distributions to peak more strongly near $90^{\circ}$ pitch angles during enhancements in the magnetic field strength than during depressions in the magnetic field strength.

The bottom panel of Fig. 4 presents results from the fluid dynamic test for the mirror instability, Eq. (1), where a negative value indicates instability. The criterion is only satisfied during sporadic intervals of weak magnetic field strength and enhanced plasma pressure after 09:40 UT (grey shading), when the amplitude of the oscillations also increases.

This is similar to results obtained in the past for waves attributed to the drift mirror instability. Zhu and Kivelson (1994) and Vaivads et al. (2001) also reported that the instability was only satisfied during intermittant intervals whereas Lanzerotti et al. (1969); Takahashi et al. (1987b); Baumjohann et al. (1987); Woch et al. (1988) presented events which did not satisfy the instability. However, Rae et al. (2007) analyzed Equator-S observations indicating that the criterion was satisfied throughout most of an extended $(\sim 7 \mathrm{~h})$ interval at radial distances up to $L \sim 11$ in the dawnside magnetosphere. As shown in some theoretical papers (Pokhotelov et al., 1986; Cheng and Lin, 1987; Woch et al., 1988), incorporating the effects of a more realistic magnetic field geometry such as a non-bi-Maxwellian velocity distribution or the coupling with other modes may reduce the instability threshold. Hellinger (2008) reported that the threshold condition for the drift mirror instability in the case of one cold species was incorrect and it was difficult to get it analytically in this case.

The unstable wave propagates at the ion drift velocity which in turn depends upon the direction of the pressure gradient. In our case, the gradient points inward. Using the finite gyroradius technique, we determined a $10 \mathrm{~km} \mathrm{~s}^{-1}$ sunward velocity for the waves. This velocity represents the sum of the particle drift velocities and the background $\boldsymbol{E} \times \boldsymbol{B}$ convection. Using observed values for the magnetic field strength and pressure as a function of radial distance, we can estimate the (antisunward) magnetic field and pressure gradient drifts to be on the order of 15 and $1 \mathrm{~km} \mathrm{~s}^{-1}$, respectively. If so, the sunward $\boldsymbol{E} \times \boldsymbol{B}$ convection velocity must be on the order of $25 \mathrm{~km} \mathrm{~s}^{-1}$. This requires an electric field of $0.5 \mathrm{mV} / \mathrm{m}$ which seems reasonable for this quiet day. Our results are consistent with those of Takahashi et al. (1987c), who reported, that the compressional waves move westward in the plasma rest frame.

Finally, we expect ion pitch angle distributions to peak more strongly near $90^{\circ}$ pitch angles during magnetic field strength enhancements than during magnetic field strength depressions. Inspection of the normalized 5-10 keV ion pitch angle distributions shown in Fig. 7 confirm that this was indeed the case. 


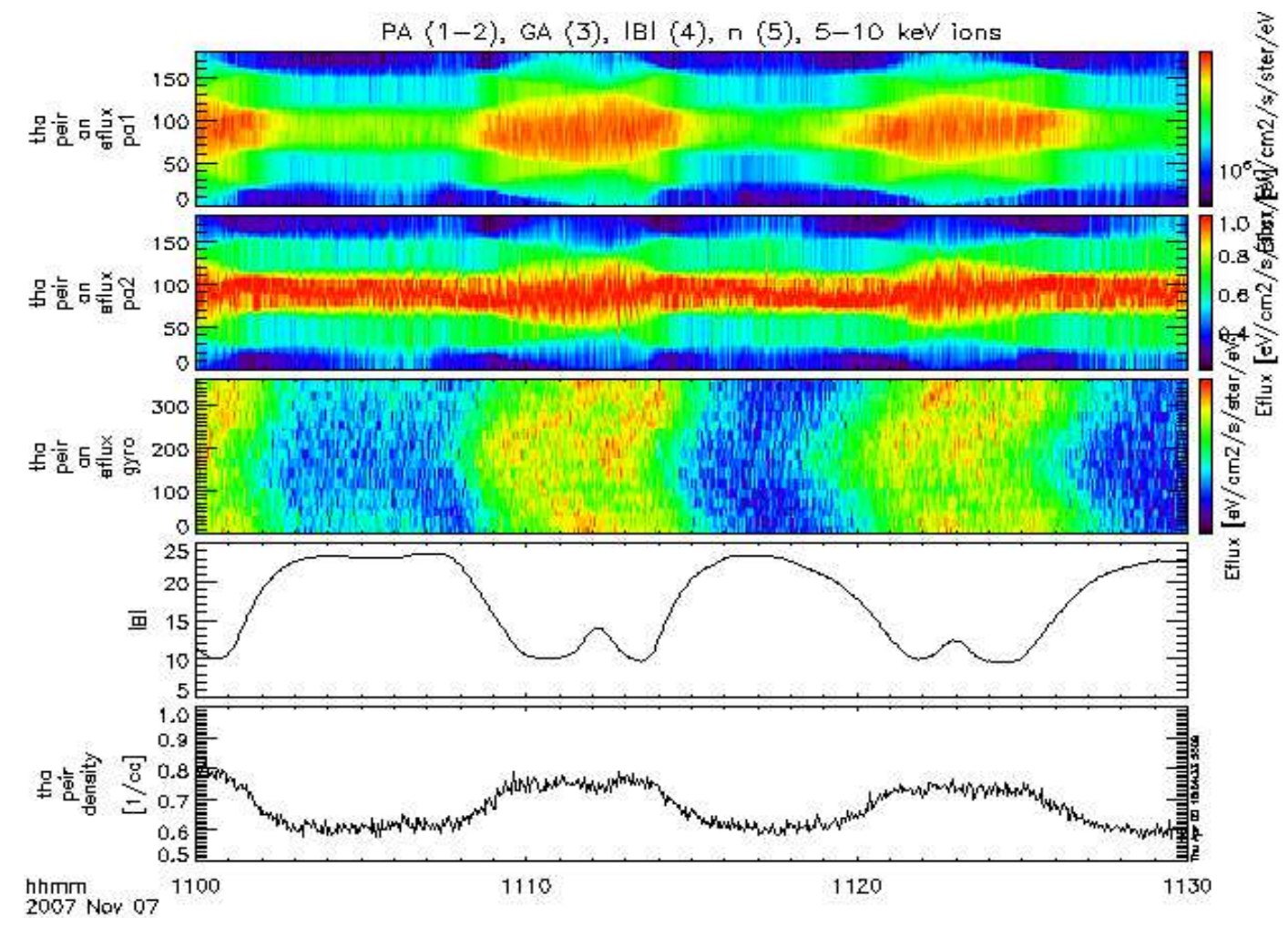

Fig. 7. Particle, magnetic and plasma data from 11:00 UT to 12:24 UT on 7 November 2007 observed by THEMIS-A. From top to bottom, the panels show the $5-10 \mathrm{keV}$ ion pitch angle distribution, the $5-10 \mathrm{keV}$ ion pitch angle distribution normalized to the peak flux during each spin, the $5-10 \mathrm{keV}$ ion gyrophase distribution integrated over pitch angles $45^{\circ}-135^{\circ}$, the magnetic field magnitude, and the ion number density versus time.

We conclude that although the instability criteria were only sporadically satisfied, the main properties of observations on 7 November 2007 are consistent with an interpretation of the waves in terms of the drift-mirror instability.

\section{Conclusions}

We used high-time resolution THEMIS-A magnetic and plasma data to determine the characteristics of compressional pulsations seen in the dawnside magnetosphere at distances of 10.5 to $13 R_{E}$ from Earth on 7 November 2007. In contrast to previous reports, pulsation periods slowly decreased, but amplitudes slowly increased, over this radial distance. We showed that the sum of the magnetic and plasma pressures was maintained during the pulsations, i.e. that they oscillated in antiphase.

Ion temperatures were greater perpendicular than parallel to the magnetic field. By contrast, electron temperatures were almost isotropic. Electron temperatures were small, but not negligible, by comparison with ion temperatures. They must be used for accurate calculation of the mirror mode instability criteria.
We inferred the motion of the wave fronts from the finite gyroradius effects seen in observations of $5-10 \mathrm{keV}$ ions, whose fluxes peaked near $90^{\circ}$ pitch angles. The waves generally move sunward at velocities $\sim 10 \mathrm{~km} \mathrm{~s}^{-1}$ with the background plasma convection flow or antisunward in the rest plasma frame. Wavelengths $\lambda$ ranged from 6700 to $23300 \mathrm{~km}$, indicating azimuthal numbers $m$ in the range from 18 to 76. The characteristics of the waves discussed in this paper are consistent with those for compressional pulsations determined in previous studies.

We considered three possible explanations for the waves: a drift-bounce instability, substorm injection, and the driftmirror instability. We found that particles with low energies of $69-628 \mathrm{eV}$ or high energies of $28-615 \mathrm{keV}$ would have been required to account for drift-bounce resonance during this interval, but inspection revealed only background fluxes of particles with these energies. Nor was there any evidence for substorm injections of suprathermal ions or abrupt enhancements in convection.

We tested the mirror instability criterion for the interval from 08:00 UT to 12:24 UT and found that it was satisfied during sporadic intervals of weak magnetic field strength and enhanced plasma pressure after 09:40 UT. Consistent with an interpretation in terms of drift mirror mode waves, plasma 
THEMIS A

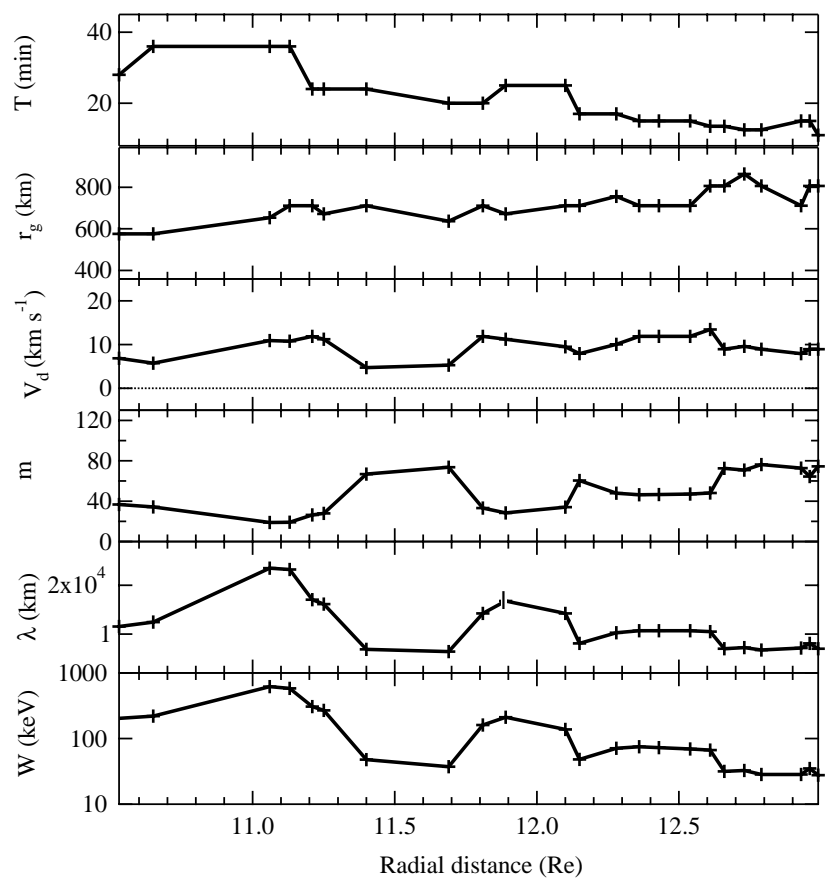

Fig. 8. Particle and wave parameters from 08:00 UT to 12:24 UT on 7 November 2007 for THEMIS-A. From top to bottom, the panels show the wave period, proton Larmor radius, wave phase velocity, azimuthal wave number, wavelength and energy of resonant driftbounce ions versus radial distance.

and magnetic pressures varied in antiphase, the waves moved sunward at speeds similar to those predicted by the theory, and ion pitch angle distributions were anisotropic and peaked more strongly near $90^{\circ}$ pitch angles during magnetic field strength enhancements than during magnetic field strength depressions. Further observational and theoretical development of theory is required to shed light on the real nature of the driving mechanism of compressional pulsations.

Acknowledgements. THEMIS is supported by NASA NAS502099. We are grateful to the ESA, FGM, and SST teams for supplying the THEMIS software and data, which we obtained in part from NASA's CDAWeb service. We thank M. Connors for providing ground magnetometer data and useful suggestions. We also thank J. Rae and I. Mann for helpful comments. Work at GSFC was supported by the THEMIS project, while work by G. I. K. at the University of Maryland was supported by a grant from NASA/GSFC NNX08AD94G.

Topical Editor I. A. Daglis thanks O. Pokhotelov and J. Rae for their help in evaluating this paper.

\section{References}

Anderson, B. J., Engebretson, M. J., Rounds, S. P., et al.: A statistical study of Pc3-5 pulsations observed by the AMPTE/CCE magnetic field experiment:1. Occurrence distributions, J. Geophys. Res., 95, 10495-10523, 1990.

Angelopoulos, V.: The THEMIS mission, Space Sci. Rev., 141, 534, doi:10.1007/s11214-008-9336-1, 2008.

Auster, U., Glassmeier, K.-H., Rounds, S. P., et al.: The THEMIS Fluxgate Magnetometer, Space Sci. Rev., 141, 235264, doi:10.1007/s11214-008-9365-9, 2008.

Barfield, J. N. and McPherron, R. L.: Statistical characteristics of storm-associated Pc5 micropulsations observed at synchronous equatorial orbit, J. Geophys. Res., 77, 4720-4733, 1972.

Baumjohann, W., Sckopke, N., LaBelle, J., et al.: Plasma and field observations of a compressional Pc 5 wave event, J. Geophys. Res., 92, 12203-12212, 1987.

Chandrasekhar, S., Kaufman, A. N., and Watson, K. M.: The stability of the pinch, Proc. Roy. Soc. (London), 245A, 435-456, 1958.

Chen, L. and Hasegawa, A.: Kinetic theory of geomagnetic pulsations.1. Internal excitations by energetic particles., J. Geophys. Res., 96, 1503-1512, 1991.

Cheng, C. Z. and Lin, C. S.: Eigenmode analysis of compressional waves in the magnetospere, Geophys. Res. Lett., 8, 884-887, 1987.

Coleman Jr., P. J.: Geomagnetic storms at ATS 1, in: Intercorrelated Satellite observations related to solar events, edited by: Manno, V. and Page, D. E., p. 251, D. Reidel, Hingham, Mass., 1970.

Constantinescu, O. D., Glassmeier, K.-H., Plaschke, F., et al.: THEMIS observations of dusk side compressional Pc 5 pulsations, J. Geophys. Res., 114, A00C25, doi:10.1029/2008JA013519, 2009.

Hamlin, D. A., Karplus, R., Vik, R. C., and Watson, K. M.: Mirror and azimuthal drift frequencies for geomagnetically trapped particles, J. Geophys. Res., 66, 1-4, 1961.

Hasegawa, A.: A drift mirror instability in the magnetosphere, Phys. Fluids, 12, 2642-2650, 1969.

Haerendel, G., Baumjohann, W., Georgescu, E., Nakamura, R., Kistler, L. M., Klecker, B., Kucharek, H., Vaivads, A., Mukai, T., and Kokubun, S.: High-beta plasma blobs in the morningside plasma sheet, Ann. Geophys., 17, 1592-1601, 1999, http://www.ann-geophys.net/17/1592/1999/.

Haerendel, G., Georgescu, E., Glassmeier, K. H., Klecker, B., Bogdanova, Y. V., Rème, H., and Frey, H.: Cluster observes formation of high-beta plasma blobs, Ann. Geophys., 22, 2391-2401, 2004 , http://www.ann-geophys.net/22/2391/2004/.

Hellinger, P.: Comment on the drift mirror instability, Phys. Plasmas, 15, 054502, doi:10.1063/1.2912961, 2008.

Kivelson, M. G. and Southwood, D. J.: Charged particle behaviour in low-frequency geomagnetic pulsations, 4. Compressional waves, J. Geophys. Res., 90, 1486-1498, 1985.

Kremser, G., Korth, A., Feier, J. A., Wilken, B., Gurevich, A. V., and Amata, E.: Observations of quasi-periodic flux variations of energetic ions and electron associated with Pc5 geomagnetic pulsations, J. Geophys. Res., 86, 3345-3356, 1981.

Kokubun, S., Kivelson, M. G., McPherron, R. L., et al.: OGO 5 observations of Pc 5 waves: Particle flux modulations, J. Geophys. Res., 82, 2774-2786, 1977. 
Lanzerotti, L. J., Hasegawa, A., and Maclennan, C. G.: Drift mirror instability in the magnetosphere: Particle and field oscillations and electron heating, J. Geophys. Res., 74, 5565-5578, 1969.

Lessard, M. R., Hudson, M. K., and Lühr, H.: A statistical study of Pc 3-Pc 5 magnetic pulsations observed by the AMPTE/Ion Release Module Satellite, J. Geophys. Res., 104, 4523-5638, 1999.

Lin, C. S. and Parks, G. K.: The coupling of Alfven and compressional waves, J. Geophys. Res., 83, 2628-2636, 1978.

Lin, N., McPherron, R. L., and Kivelson, M. G.: An unambiguous determination of the propagation of a compressional Pc5 wave, J. Geophys. Res., 93, 5601-5612, 1988.

Nagano, H. and Araki, T.: Long-duration Pc5 pulsations observed by geostationary satellites, Geophys. Res. Let., 10, 908-911, 1983.

McFadden, J. P., Carlson, C. W., Larson, D., et al.: The THEMIS ESA Plasma Instrument and In-flight Calibration, Space Sci. Rev., 141, 277, doi:10.1007/s11214-008-9440-2, 2008.

Pokhotelov, O., Pilipenko, V., and Amata, A. E.: Drift anisotropy instability of a finite $\beta$ magnetospheric plasma, Planet. Space Sci., 33, 1229-1241, 1985.

Pokhotelov, O., Pilipenko, V. A., Nezlina, Y. M., et al.: Excitation of high $\beta$ plasma instabilities at the geostationary orbit: Theory and observations, Planet. Space Sci., 34, 695-712, 1986.

Rae, I. J., Mann, I. R., Watt, C. E. J., et al.: Equator-S observations of drift mirror waves in the dawnside magnetosphere, J. Geophys. Res., 112, A11203, doi:10.1029/2006JA012064, 2007.

Southwood, D. J., Dungey, J. W., and Etherington, R. J.: Bounce Resonance interaction between pulsations and trapped particles, Plan. Space Sci., 17, 349-361, 1969.

Southwood, D. J.: The behavior of ULF waves and particles in the magnetosphere, Planet. Space Sci., 21, 53-65, 1973.

Southwood, D. J.: A general approach to low-frequency instability in the ring current plasma, J. Geophys. Res., 81, 3340-3348, 1976.

Southwood, D. J.: Low frequency pulsation generation by energetic particles, J. Geomagn. Geoelectr., Suppl., II, 32, 75-88, 1980.

Southwood, D. J. and Kivelson, M. G.: Charged particle behavior in low-frequency geomagnetic pulsations. 1. Transverse waves, J. Geophys. Res., 86, 5643-5655, 1981.

Southwood, D. J. and Hughes, W. J.: Theory of hydromagnetic waves in the magnetosphere, Space Sci. Rev., 35, 301-366, 1983.

Southwood, D. J. and Kivelson, M. G.: Mirror instability: 1. Physical mechanism of linear instability, J. Geophys. Res., 98, 91819187, 1993.

Southwood, D. J. and Kivelson, M. G.: Frequency doubling in ultralow frequency wave signals, J. Geophys. Res., 102, 2715127158, 1997.

Su, S.-Y., Konradi, A., and Fritz, T. A.: On propagation direction of ring current proton ULF waves observed by ATS 6 at $6.6 \mathrm{Re}, \mathrm{J}$. Geophys. Res., 82, 1859-1867, 1977.
Su, S.-Y., McPherron, R. L., Konradi, A., and Fritz, T. A.: Observations of ULF Oscillations - in the Ion Fluxes at Small Pitch Angles with ATS 6, J. Geophys. Res., 85, 515-522, 1980.

Takahashi, K., Higbie, P. R., and Baker, D. N.: Azimuthal propagation and frequency characteristic of compressional Pc 5 waves observed at geostationary orbit, J. Geophys. Res., 90, 1473$1485,1985$.

Takahashi, K. and Higbie, P. R.: Antisymmetric standing wave structure associated with the compressional Pc 5 pulsation of November 14, 1979, J. Geophys. Res., 91, 11163-11178, 1986.

Takahashi, K., Zanetti, L. J., Potemra, T. A., et al.: A model for the harmonic of compressional Pc5 waves, Geophys. Res. Let., 14, 363-366, 1987a.

Takahashi, K., Fennell, J. F., Amata, E., et al.: Field-aligned structure of the storm time Pc5 wave of November 14-15, 1979, J. Geophys. Res., 92, 5857-5864, 1987b.

Takahashi, K., Lopez, R. E., McEntire, R. W., et al.: An easward propagating compressional Pc5 wave observed by AMPTE/CCE in the postmidnight sector, J. Geophys. Res., 92, 13472-13484, 1987c.

Takahashi, K., Cheng, C. Z., McEntire, R. W., and Kistler, L. M.: Observation and theory of Pc5 waves with harmonically related transverse and compressional components, J. Geophys. Res., 95, 977-989, 1990a.

Takahashi, K., McEntire, R. W., Lui, A. T. Y., and Potemra, T. A.: Ion flux oscillations associated with a radially polarized transverse Pc5 magnetic pulsation, J. Geophys. Res., 95, 3717-3731, 1990b.

Vaivads, A., Haerendel, G., Baumjohann, W., et al.: Compressional Pc5 pulsations as sloshing in the plasma sheet, J. Geophys. Res., 105, 23287-23292, 2000.

Vaivads, A., Baumjohann, W., Haerendel, G., et al.: Compressional Pc5 type pulsations in the morning plasma sheet, Ann. Geophys., 145(9), 311-320, 2001.

Woch, J., Kremser, G., Korth, A., Pokhotelov, O. A., Pilipenko, V. A., and Nezlina, Yu. M.: Curvature-driven drift mirror instability in the magnetosphere, Planet. Space Sci., 36, 383-392, 1988.

Woch, J., Kremser, G., and Korth, A.: A comprehensive investigation of compressional ULF waves observed in the ring current, J. Geophys. Res., 95, 15113-15132, 1990.

Walker, A. D. M., Greenwald, R. A., Korth, A., et al.: STARE and GEOS 2 observations of a storm time Pc5 ULF pulsation, J. Geophys. Res., 87, 9135-9146, 1982.

Zhu, X. M. and Kivelson, M. G.: Compressional ULF waves in the outer magnetosphere: 1. Statistical study. J. Geophys. Res., 96, 19451-19467, 1991.

Zhu, X. M. and Kivelson, M. G.: Compressional ULF waves in the outer magnetosphere: 2 . A case study of Pc5 type wave activity, J. Geophys. Res., 99, 241-252, 1994. 